\title{
Operating Margins For A Pulse-Driven Programmable Voltage Standard
}

\author{
S. P. Benz, C. J. Burroughs, and C.A. Hamilton \\ National Institute of Standards and Technology \\ 325 Broadway, Boulder, CO 80303-3328 USA
}

\begin{abstract}
We have designed and fabricated a Josephson voltage standard where the voltage can be rapidly and continuously programmed by changing the repetition frequency of a pulse drive. Simulations are made to optimize the operating margins of the circuit for different pulse waveforms. The response of a 1000-junction array of $\mathrm{Nb}-\mathrm{PdAu}-\mathrm{Nb}$ junctions is measured, and constantvoltage step heights are characterized as a function of the pulse amplitude, pulse width, and frequency. A dc bias range of $0.62 \mathrm{~mA}$ is demonstrated over a continuous voltage-tunable range from $-6.2 \mathrm{mV}$ to $+6.5 \mathrm{mV}$.
\end{abstract}

\section{INTRODUCTION}

NIST is developing accurate and fast programmable voltage standards for calibration of dc reference standards and digital voltmeters, characterization of commercial D/A and A/D converters, and generation of digitally synthesized ac waveforms with calculable RMS voltages. In the latter application, primary standards for ac voltage presently rely on thermal voltage converters that compare the heating effect of ac and dc inputs [1]. Direct waveform synthesis from a Josephson source would provide the first independent check on the accuracy of thermal voltage converters. A Josephson programmable voltage standard is essentially a D/A converter, with output voltage steps that have the full accuracy of the Josephson representation of the SI volt.

Two different programmable voltage standard designs are under development in order to realize these metrology applications [2]-[5]. The first design uses a sinusoidallydriven series array of Josephson junctions divided into a binary sequence of arrays [2]-[4]. This device has the potential to achieve voltages of $1-10 \mathrm{~V}$ with a resolution of 20-30 $\mu \mathrm{V}$. However, since bias currents are used to switch each array between constant voltage steps, the accuracy of generated ac waveforms is limited by the speed of the semiconductor current drivers and other bias delays. Recently, we have proposed a new type of programmable voltage standard that uses an array of junctions driven by pulses [5]. By using a pulse drive instead of a sinusoidal drive, frequency can be used to change the array voltage instead of the number of junctions. Pulse-driven arrays have the potential for providing standards with improved accuracy over broad ranges of frequency and amplitude, without inaccuracies imposed by switching transients. This paper describes the first experimental results on arrays specifically designed for the pulse-driven programmable voltage standard application.

Manuscript received August 25, 1996. This research is supported in part by the U.S. Army as CCG Project \#346.

Contribution of the U.S. Government, not subject to U.S. copyright.

\section{A. Pulse Shape}

\section{SIMULATIONS}

Simulations have been performed for the waveform shown in Fig. 1(a) using both cosine (solid) and square (dashed) pulse shapes. The pulse is defined by its width $\tau$ (full width at half maximum for the cosine-shaped pulse) and current amplitude $I_{p}$. The repetition frequency $f$ determines the separation period $T=1 / f$ between the pulses. The Josephson junction achieves its voltage accuracy by converting the input pulses to quantized voltage pulses whose time integral is equal to a single flux quantum $\mathrm{h} / 2 \mathrm{e} \approx 2.07 \mathrm{mV} \cdot \mathrm{ps}$. When a periodic waveform is applied, the time-averaged voltage across the junction will itself be quantized, having constant voltage steps at voltages proportional to the repetition frequency $V=n f / K_{J}$, where $n$ is the step number related to the number of quantized pulses, or phase slips, from the junction that are generated for each input pulse. $K_{J}=483$ $597.9 \mathrm{GHz} / \mathrm{V}$ is the Josephson constant. We define a normalized inverse pulse width, $P=1 /\left(2 \tau f_{c}\right)$, and normalized repetition frequency, $\Omega=f / f_{c}$, where $f_{c}=I_{c} R K_{J}$ is the junction characteristic frequency, $I_{C}$ is the junction critical current, and $R$ is its resistance. Whereas frequency determines the step voltage, the pulse width determines the maximum step height $\Delta I_{n}$ [5]. Step heights approach $2 I_{c}$ for $P \gg 1$ and are on the order of $I_{c}$ for $P=1$. The primary advantage of the pulse drive is that the step heights are nearly independent of frequency from zero to the maximum $\Omega=P$. In comparison, the step heights for a sinusoidal drive fall to zero for frequencies below $\Omega / 3$.

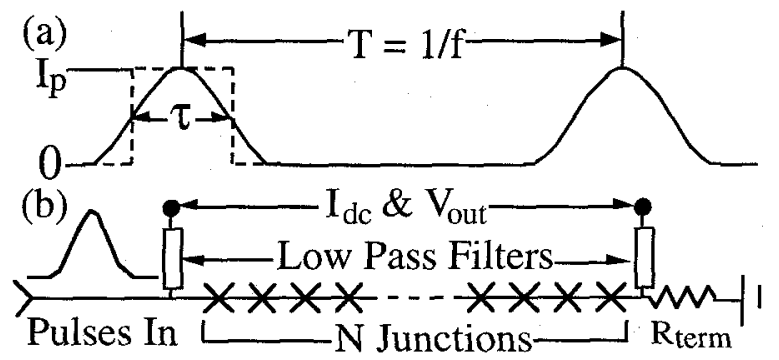

Fig. 1. (a) Periodic pulse waveform for cosine and square pulse shapes. (b) Schematic array circuit.

A comparison was made between the square- and cosineshaped pulses that showed only minor differences in the step heights as a function of pulse amplitude and width. The simulation method used to obtain the constant voltage step boundaries is described elsewhere [6]. For example, when the $\mathrm{dc}$ bias range of the step is centered at zero current (zerocentered), the step heights are slightly smaller for squareshaped pulses when $P<1$, and slightly lower pulse amplitudes are needed to center the steps for square pulses 
having widths near $P=1$. These minor differences are probably due to the higher harmonic content associated with the square-shaped pulse. Inspection of the Fourier transforms of the cosine and square-shaped waveforms shows that most of the harmonic content of both waveforms occurs over the same range, primarily for harmonic numbers $m \leq T / \tau$, or frequencies $m \Omega \leq 2 P$. In summary, we find that the step heights are determined primarily by pulse width, essentially independent of pulse shape, provided that widths are defined (as in Fig. 1(a)) to have the same dc component $I_{p} \tau /$.

\section{B. Operating Margin}

For complex waveform generation, it is important to know the current boundaries of the steps for different patterns (words) of pulses applied to the array in a repetitive fashion. For example, a 10-bit word could result in different bias boundaries depending on the placement of 1's (pulses) and 0's (no pulses) in the word. We examined the step boundaries of a single junction by using different word patterns, such as 1001110000 , with a 10-bit length, such that a pulse is sent to the array for each 1 and no pulse was sent for each 0 . We find that the operating margin is determined by the two extreme cases of a single one (1000000000) and all ones (111111111). These two cases correspond to the $\Omega=0.1$ and 1.0 repetition frequencies. Figure 2 shows the dc bias boundaries of the $n=1$ step for these two cases as a function

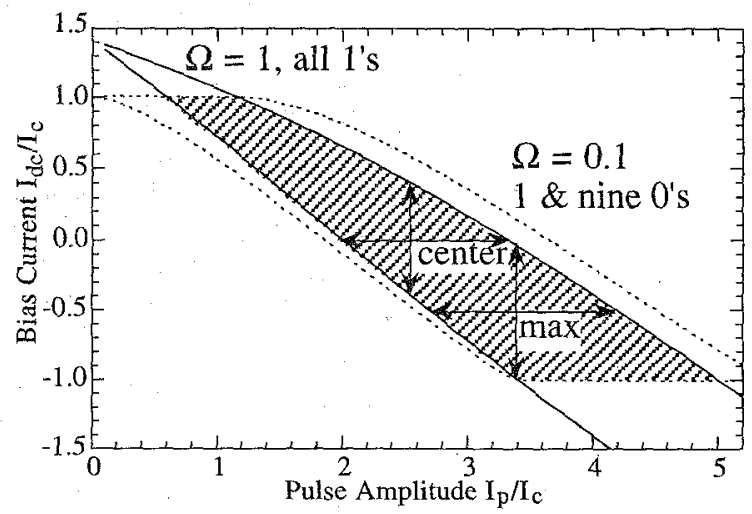

Fig. 2. Bias range vs. pulse amplitude showing operating margins for $P=1$.

of pulse amplitude at $P=1$. The shaded region indicates the intersection of these two cases and thus determines the operating margins for arbitrary word patterns. The zerocentered operating margin is determined by the $\Omega=1$ boundaries yielding a centered step height $I_{d c}=(0 \pm 0.397) I_{c}$ and $I_{p}=(2.55+0.75,-0.55) I_{c}$. The operating point with the largest bias range is determined by the intersection of the two lower boundaries and occurs at $I_{p}=(3.40+0.83$, $-0.68) I_{c}$ and $I_{d c}=(-0.522 \pm 0.476) I_{c}$. These wide margins for both pulse amplitude and dc bias are sufficient to drive typical loads found in metrology applications.

For metrology applications it is desirable to have symmetric output waveforms with both positive and negative voltages. Negative voltages can be made by switching the sign of the pulse amplitude. We are primarily interested in zero-centered steps, because dc current offsets required to center the bias in its optimum operating range would also need to change sign for negative voltages. Switching of such offsets will limit the speed and accuracy as in the sinusoidally-driven programmable voltage standard. The bias range of the zero-centered step $\left(\approx 0.8 I_{c}\right)$ is only $17 \%$ smaller than the maximum step height. Since the maximum step requires an undesirable negative offset, we focus our operating margin analysis on zero-centered steps and $P=1$ pulse widths which provide sufficiently large step heights for a reasonable pulse amplitude [5].

\section{EXPERIMENTS}

\section{A. Circuit Design}

The two most important requirements for the pulse-driven programmable voltage standard are junction uniformity and power uniformity over the whole array of junctions. NIST has been developing superconductor-normal-superconductor (SNS) junctions made from Nb-PdAu-Nb trilayers [3]-[5], [7] for standards applications. The fabrication process for the $4 \mu \mathrm{m} \times 4 \mu \mathrm{m}$ junctions used here is described in detail elsewhere [7]. Arrays of these junctions have shown uniformity and run-to-run reproducibility that are sufficient for our applications [4], [7]. Power uniformity for sinusoidally-driven arrays has been achieved through the use of single quarter-wavelength sections that make the bias leads appear as open circuits at the drive frequency [4], [ 7]. The center frequency of these stop band filters is set to the design frequency of the array.

For the pulse-driven arrays, the bias lead filters must not attenuate the low frequency output waveform and at the same time they must appear as open circuits at the array transmission line over the full range of drive frequencies. Thus, the filters should be low pass with a stop band above a cutoff frequency that is determined by the maximum required output bandwidth $f_{\text {out }}<f_{\text {cutoff }}$ [5]. The flat stop band should have uniform attenuation over most if not all of the frequency range from $\Omega_{\text {cutoff }}$ to $2 P$.

In order to measure the step height dependence on pulse amplitude, pulse width, and repetition frequency, we have fabricated arrays using circuits with two simple filter designs. The first circuit uses the same quarter-wavelength filters described above. These filters, although not ideal, can cover a significant portion of the harmonic content by centering them at frequency $P$. The schematic circuit for this design is shown in Fig. 1(b). The filters used in this experiment are $1 \mathrm{~cm}$ long, resulting in a center frequency of $3 \mathrm{GHz}$; and are described in detail in [7]. The second circuit replaces each band stop filter in Fig. 1(b) with a pair of $50 \Omega$ lumped resistors to separate output terminals. This makes possible four-point current-voltage measurements of the array.

The 1000-junction arrays are distributed along the center conductor of a $50 \Omega$ coplanar transmission line that is terminated with a matched resistor. Capacitors cannot be used along the pulse propagation path because the dc component of the pulse waveform is essential for biasing the array [5], [7]. For high critical current arrays, dc heating in the bias resistors prevented some measurements, so we present results for both circuits and for arrays with different critical currents. 


\section{B. Pulse Width and Amplitude}

The data shown in Fig. 3 are from an array with $3 \mathrm{GHz}$ stop band filters. The junctions have $I_{c}=1.45 \mathrm{~mA}$ and $R=$ $1.50 \mathrm{~m} \Omega$ resulting in a characteristic frequency of $1.05 \mathrm{GHz}$. The pulse source generates square pulses with 60 ps transitions over repetition frequencies from $33 \mathrm{MHz}$ to 3.5 $\mathrm{GHz}$. The dc bias boundaries and step heights as a function of pulse amplitude are shown in Figs. 2(a) and (b) for the $n=$ 0,1 and 2 steps. The pulse width is $475 \mathrm{ps}(P=1)$ and the repetition frequency is $105 \mathrm{MHz}(\Omega=0.1)$. The experimental points are plotted against simulations (solid lines) of a single junction driven with square pulses for the

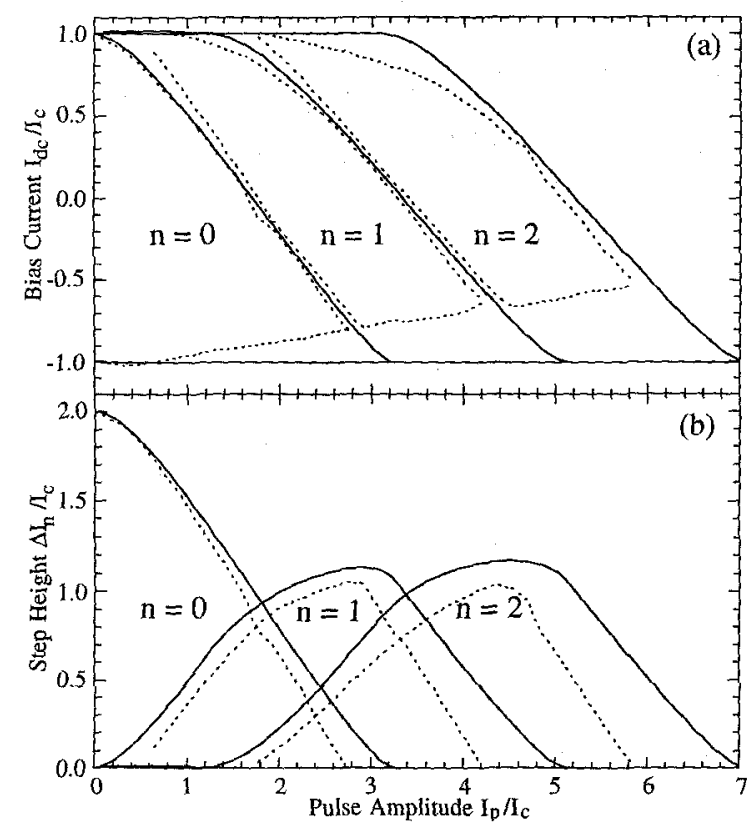

Fig. 3. DC bias boundaries (a) and step heights (b) vs. puise amplitude. $T=$ $475 \mathrm{ps}(P=1)$ and $f=105 \mathrm{MHz}(\Omega=0.1)$. Solid lines show simulated results.

same reduced frequencies. The experimental pulse amplitude through the array, $I_{p}$, is approximated by $I_{p}=\alpha V_{p} /(1000 R+$ $\left.R_{\text {term }}\right)$, where $V_{p}$ is the voltage amplitude from the pulse generator, $R_{\text {term }}$ is the termination resistance, and $\alpha$ is the fraction of current transmitted from the pulse generator to the array. The termination resistance was measured to be 55.6 $\Omega$, so that $I_{p}=\alpha V_{p} / 57.1 \Omega$. The experimental data are within the simulation boundaries when $\alpha$ is chosen to be 0.85 .

The experimental data fit well to the simulation data. Some deviation from the simulation may be due to junction nonuniformity and nonideal pulses with $60 \mathrm{ps}$ transitions and ringing following the transitions. However, the primary cause is probably due to pulse waveform nonuniformity, in both amplitude and phase, resulting from the non-ideal microwave circuit. Although better filters would likely improve these results, the zero-centered $n=1 \mathrm{step}$ boundaries are sufficient $(>1 \mathrm{~mA}$ ) for operation in the programmable voltage standard.

Next we explored the dependence of the zero-centered $n=1$ step on pulse width. We investigated the same $3 \mathrm{GHz}$ filtered circuit described above as well as the resistively biased circuit from the same $1 \mathrm{~cm} \times 1 \mathrm{~cm}$ chip. The resistively biased circuit shows slightly more uniform results, so we present these data. The junctions have $I_{c}=1.28 \mathrm{~mA}$ and $R=1.45$ $\mathrm{m} \Omega$ resulting in a characteristic frequency of $895 \mathrm{MHz}$. Figures 4(a) and (b) show the zero-centered step height and pulse amplitude required to center the step as a function of

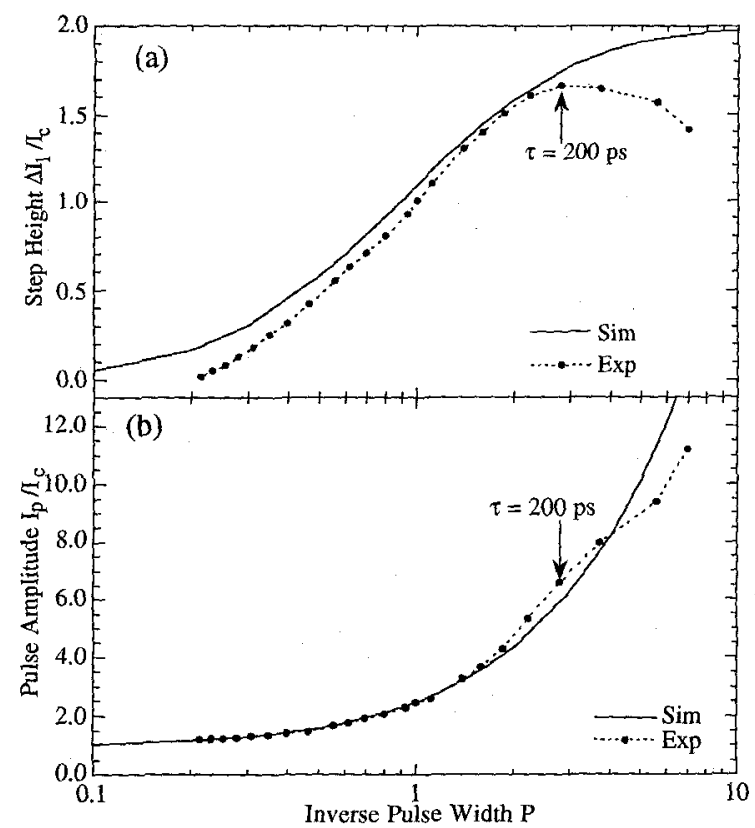

Fig. 4. $n=1$ step height and pulse amplitude required to center the step vs. inverse pulse width. Solid lines show simulated results.

inverse pulse width. The repetition frequency is set to 35 $\mathrm{MHz}$ for all data points. The simulation data presented are for a square pulse shape. Except for the four smallest pulse widths ( $\tau \leq 200 \mathrm{ps}$ ), the experimental data follow the simulation data fairly well. The poor fit for the four largest values of $P$ (smallest $\tau$ ), are due to distortions in the waveform from the pulse generator. The distortions were determined by inspection of the waveform on a high-speed digital oscilloscope. The smaller step heights for small $P$ may be due to noise, since the step heights are decreasing as $P$ decreases.

\section{Repetition Frequency}

Finally, we focus on the repetition frequency which is used to change the output voltage in a pulse-driven programmable voltage standard. It is the prediction that the step boundaries are nearly independent of frequency that makes the pulsedriven arrays ideal for ac waveform synthesis [5]. Figure 5 shows the first experimental data to confirm this feature of pulse-driven arrays over a wide range of repetition frequencies. The measurements are performed on the same resistive biased circuit as above, but from a different chip on the same wafer $\left(I_{c}=1.13 \mathrm{~mA}\right.$ and $R=1.41 \Omega$ ). The dc bias range of the $n=1$ step is plotted as a function of the array step voltage $\left(1000 f / K_{l}\right)$. The frequency is stepped from $40 \mathrm{MHz}$ to $3 \mathrm{GHz}$ in increments of $20 \mathrm{MHz}$. The pulse width is $160 \mathrm{ps}(P \approx 4)$ 
and the pulse amplitude $(1.55 \mathrm{~V})$ is adjusted to center the step at low frequencies. A $10 \mathrm{~dB} \mathrm{dc}-18 \mathrm{GHz}$ attenuator is used to reduce the pulse amplitude before the signal enters the cryoprobe. The boundaries of the step are chosen when the array voltage exceeds a $\pm 10 \mu V$ threshold on either side of the target voltage. Negative voltages are generated by using negative pulse amplitudes. The operating region of the system was limited by frequency dependence in the amplitude and voltage offsets from the commercial pulse generator. We corrected the frequency-dependent offsets by measuring the dc voltage across the termination resistor and adding a compensating voltage at each frequency. Even without corrections for the frequency-dependent amplitude variation, we can maintain a total bias range of $0.54 \mathrm{~mA}$ over a voltage

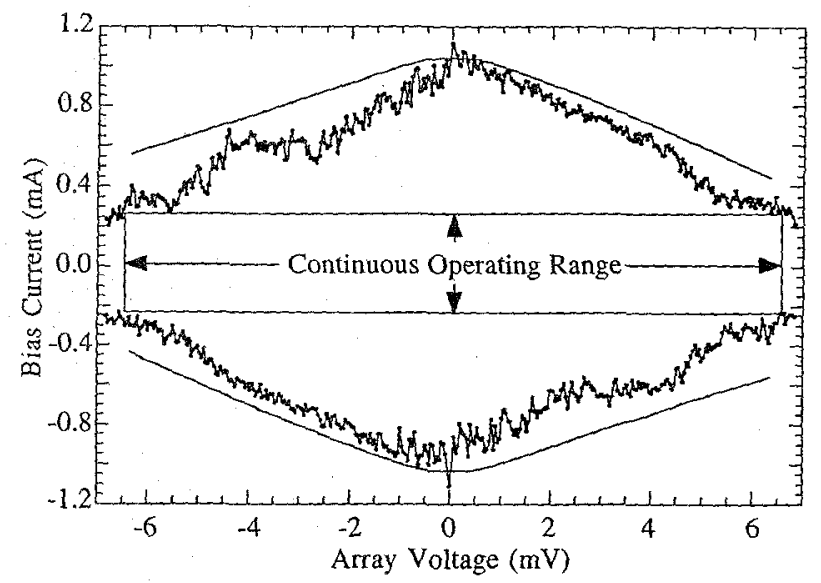

Fig. 5. DC bias boundary vs. array $n=1$ step voltage. Solid lines show the simulated boundary with $P=4$ and $I_{p}=8.27 I_{c}$ for zero-centered steps.

range from $-6.5 \mathrm{mV}$ to $+6.5 \mathrm{mV}$, corresponding to the maximum square-wave frequency of $3.13 \mathrm{GHz}$. Although this array has $50 \Omega$ resistors on the bias leads, arrays with 3 $\mathrm{GHz}$ bias filters show similar behavior. Despite the nonlinearities of the pulse generator, this array could be used as a waveform generator with $13.0 \mathrm{mV}$ peak-to-peak output.

\section{Waveform Synthesis}

Using the same circuit without correcting the pulse waveform for voltage offsets, we achieved an operating region with a bias range of $0.7 \mathrm{~mA}$ for frequencies up to $2.4 \mathrm{GHz}$ using a pulse width of 130 ps. Figure 6 is a digital approximation to a $10.0 \mathrm{mV}$ peak-to-peak sine wave that was synthesized by driving this array with a variable frequency pulse train. The pulse train was generated using an accurately timed sequence of IEEE Bus commands to sweep the pulse repetition rate of the pulse source from 0 to $2.4 \mathrm{GHz}$ in 100 $\mathrm{MHz}$ steps. The negative half of the waveform was created by reversing the polarity of the pulse amplitude. While the curve in Fig. 6 is only an approximation to a sine wave, the important point for metrology is that its RMS value can be precisely computed. Time delays associated with the IEEE commands and the small number of junctions limited this demonstration to $77 \mathrm{mHz}$ frequency and $10 \mathrm{mV}$ peak-to-peak.

A system for generation of arbitrary waveforms at much higher frequencies would consist of a digital word generator clocked at a fixed frequency near $\Omega=1$. The Josephson junctions would quantize the input pulses and the density of ones within the word would vary according to desired voltage level at a given time in the generated waveform. The word length would determine the synthesized frequency. The

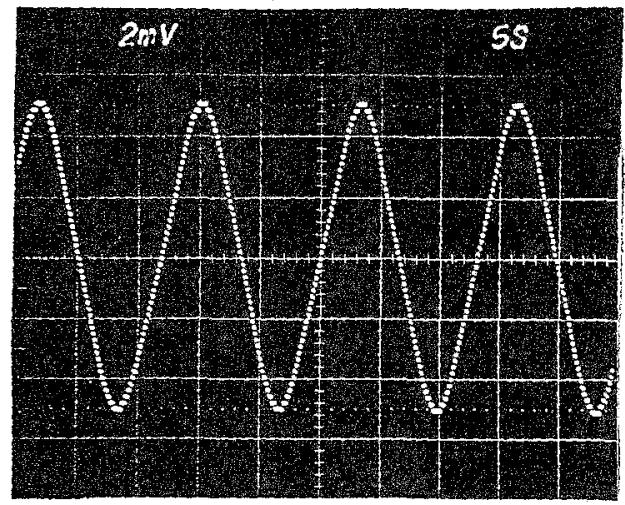

Fig. 6. Digital sine wave synthesized by a 1000 -junction array.

operating margins in section II.B are optimized for this very application. For example, using a clock frequency equal to a characteristic junction frequency, $f_{c}$, of $10 \mathrm{GHz}$, the pulse sequence $11111000001111100000 \ldots$ would create an output square wave of amplitude $N f_{c} / K_{J}$ with a frequency of $1 \mathrm{GHz}$. For a sufficiently long word length, a sinusoidal waveform would be nearly continuous compared to the discrete voltage level approximation of Fig. 4. Such precisely computable waveforms would be ideal for ac voltage metrology at frequencies up to $1 \mathrm{MHz}$.

\section{ACKNOWLEDGMENTS}

The authors thank R. L. Kautz for helpful conversations.

\section{REFERENCES}

[1] M. Klonz, "Current developments in accurate AC-DC transfer measurements," IEEE Trans. Instrum. Meas., vol. 44, pp. 363-366, April 1995.

[2] C.A. Hamilton, C.J. Burroughs, and R.L. Kautz, "Josephson D/A converter with fundamental accuracy," IEEE Trans. Instrum. Meas., vol. 44, pp. 223-225, April 1995.

[3] S.P. Benz, "Superconductor-normal-superconductor junctions for programmable voltage standards," Appl. Phys. Lett., vol. 67, pp. 2714 2716 , Oct. 1995

[4] C.A. Hamilton, S.P. Benz, C.J. Burroughs, and T. Harvey, "1-volt Josephson programmable voltage standard," IEEE Trans, Appl. Supercond., this issue, 1997.

[5] S.P. Benz and C.A. Hamilton, "A pulse-driven programmable Josephson voltage standard," Appl. Phys. Lett., vol. 68, pp. 3171-3173, May 1996.

[6] K. Wiesenfeld, S.P. Benz, and P.A.A. Booi, "Phase-locked oscillator optimization for arrays of Josephson junctions," J. Appl. Phys., vol. 76, pp. 3835-3846, Sept. 1994.

[7] S.P. Benz and C.J. Burroughs, "Constant voltage steps in Nb-PdAu-Nb Josephson junction arrays," IEEE Trans. Appl. Supercond., this issue." 